\title{
Adição à internet ou uso problemático da internet? Qual dos termos usar?
}

\author{
Beatriz Fernandes ${ }^{a}$ (iD \\ Berta Rodrigues Maia ${ }^{a^{*}}$ \\ Halley M. Pontes ${ }^{b}$ (D) \\ a Universidade Católica Portuguesa, Faculdade de Filosofia e Ciências Sociais, Centro Regional de Braga, Portugal \\ b Universidade da Tasmânia, Departamento de Psicologia. Faculdade de Medicina. Sydney, Austrália
}

\begin{abstract}
Resumo: $O$ uso da internet tem aumentado exponencialmente a nível mundial. Ainda que ele não seja por si só negativo, já que integra benefícios vários, alguns indivíduos parecem exibir problemas relacionados com o seu uso excessivo, descontrolado e disfuncional. Consequentemente, tem sido crescente, particularmente nas últimas duas décadas, o interesse dos investigadores em explorar este uso, quando excessivo e pouco saudável. Porém, e sendo um tema/constructo tratado por diferentes autores com quadros teóricos também diferentes, são vários os termos usados na literatura para descrever este fenómeno. Neste sentido, este artigo propõe-se a apresentar o trabalho uma revisão de literatura de dois dos conceitos mais usados e espartilhados na literatura científica, ou seja, adição à internet e uso problemático da internet.
\end{abstract}

Palavras-chave: adição à internet, uso problemático da internet, conceptualização.

\section{Introdução}

O uso da internet tem aumentado exponencialmente nos últimos anos, sendo cada vez mais comum. A título ilustrativo, o relatório mais recente do Instituto Nacional de Estatística (2014) dá-nos conta que cerca de 65\% da população portuguesa entre os 16 e os 74 anos usa a internet regularmente, sendo o seu uso mais prevalente entre os jovens de 16 a 24 anos. Um relatório publicado pela Marktest (2016) reportou que o número de utilizadores aumentou mais de 10 vezes nos últimos 18 anos, passando de uma penetração de 6,3\% em 1997 para 65,4\% em 2016. Já a nível mundial, mais de 7.500 milhões de pessoas têm acesso à internet, o que equivale a $49,6 \%$ da população mundial, sendo que a Europa se encontra em segundo lugar na taxa de penetração de internet, com uma percentagem de 77,4\% (Internet World Stats, 2017).

Ainda que o uso moderado e saudável da internet por si só não represente riscos significativos e seja, de modo geral, benéfico para a maioria dos utilizadores, uma minoria da população apresenta problemas relacionados ao seu uso excessivo, descontrolado e disfuncional (Pontes, Caplan, \& Griffiths, 2016). Dada sua relevância, o número de estudos publicados sobre a adição à internet tem vindo a aumentar exponencialmente no decorrer das últimas duas décadas, sendo que em 2018 mais de 1.600 estudos foram publicados em revistas científicas nacionais e internacionais (Wiederhold, 2018). De modo geral, a

* Endereço para correspondência: bmaia@braga.ucp.pt

1 Esse trabalho teve apoio de National Funds, proporcionado pela FCT (Fundação para a Ciência e a Tecnologia) para os projetos estratégicos PEst-OE/FIL/UI0683/2014. literatura científica reporta associações consistentes entre o uso problemático da internet a uma diversidade de problemas psicossociais, tais como bem-estar emocional mais pobre (cf. Griffiths, 2015; Piguet, Berchtold, Akre, $\&$ Suris, 2015; Pontes et al., 2016) ou níveis mais elevados de psicopatologia, como a depressão (e.g. Cabral, Pereira, \& Teixeira, 2018; Mendes \& Silva, 2017; Pontes, Patrão, \& Griffiths, 2014; Tokunaga \& Rains, 2016).

Ainda não existe consenso na comunidade científica em relação ao termo a utilizar para descrever o uso e abuso das novas tecnologias. Por um lado, há autores que defendem a existência de uma adição às novas tecnologias, incluída nas adições comportamentais (Carbonell, Fúster, Chamarro, \& Oberst, 2012; García del Castillo, 2013; Potenza, 2006; Vivas \& Torres, 2011; Young, 1998). Por outro, há autores que afirmam que o potencial aditivo das novas tecnologias é especulativo (Carbonell et al., 2012; Echeburúa, \& Corral, 2010; García del Castillo, 2013). Mais recentemente, vários autores têm vindo a reforçar a ideia de que o conceito de adição à internet não é adequado para descrever o fenómeno associado às consequências negativas resultantes do uso excessivo e disfuncional (Starcevic \& Aboujaoude, 2017).

Existem, por isso, diferentes modelos teóricos e terminologias utilizadas para descrever o comportamento excessivo do uso da internet, incluindo adição à internet (Young, 1998), uso patológico da internet (Davis, 2001), uso problemático da internet (Caplan, 2002), ou dependência da internet (Chen, Tarn, \& Han, 2004). Deste modo, esta revisão irá contribuir para o esclarecimento da terminologia usada nesta área, especificamente sobre os termos adição e uso problemático. 


\section{Adição à internet}

A maior parte dos trabalhos iniciais na área da adição à internet foram realizados por Kimberly Young nos Estados Unidos e Mark Griffiths no Reino Unido (e.g., Griffiths, 1995; Young, 1998). De acordo com Young (1998), a adição à internet é um termo amplo que integra uma diversidade de comportamentos e problemas de controlo dos impulsos, estando categorizados em cinco subtipos específicos: adição cibernética (uso compulsivo de sites adultos para cybersex e cyberporn), adição ao relacionamento cibernético (envolvimento excessivo em relacionamentos on-line), compulsões de rede (jogos on-line obsessivos ou compras), sobrecarga de informações (navegação excessiva) e, por último, adição ao computador (jogo de computador obsessivo).

A adição à internet tornou-se uma área relevante de investigação (King, Delfabbro, \& Griffiths, 2012); porém, atualmente, ainda não existe um consenso relativamente à definição oficial do problema e do seu lugar nas classificações, pelo facto de se tratar de um fenómeno ainda em estudo (Patrão et al., 2017) e de existirem inúmeras limitações metodológicas relacionadas com a avaliação do construto (King et al., 2012).

A adição à internet pode ser vista como uma adição especificamente psicológica (como o vício em sexo, em compras, em jogos de vídeo etc.), com particularidades comuns a outros tipos de dependência, tais como, a perda de controlo, sintomas de abstinência, forte dependência psicológica, interferência na vida diária e perda de interesse noutras atividades (Kuss \& Griffiths, 2017). A adição à internet tem sido também caracterizada como um padrão de uso mal-adaptativo que pode causar danos clinicamente significativos na vida da pessoa (Elhai, Dvorak, Levine, \& Hall, 2017). Mais recentemente, Kuss e Pontes (2019) definiram o fenómeno como um padrão comportamental envolvendo a experiência de craving disfuncional em relação ao uso da internet por períodos de tempo excessivos sem autorregulação por parte do indivíduo, resultando em significativos prejuízos psicológicos, sociais e funcionais. Ou seja, o indivíduo dependente da internet gasta um tempo total diário notável em atividades on-line que não são essenciais, desenvolvendo um afastamento dos contactos sociais fora da internet, uma distorção dos seus objetivos pessoais e dos seus interesses e uma perda do rendimento académico e/ou laboral (Patrão et al., 2017).

Para compreender melhor a adição à internet, Griffiths (2005) desenvolveu o modelo das componentes da adição que nos refere que todas as adições assentam em seis componentes comuns distintas (saliência, modificação de humor, tolerância, abstinência, conflito e recaída). Ou seja, as adições (com ou sem o uso de substância) fazem parte de um processo biopsicossocial e existem dados crescentes de que os comportamentos excessivos de todos os tipos parecem ter muitas semelhanças. Esta é uma conclusão que recebe suporte de estudos recentes que revelam semelhanças entre diversos tipos de comportamentos aditivos (com ou sem o uso de substância) a nível do circuito neural e ativação da área de reforço comportamental no cérebro (Sharifat, Rashid, \& Suppiah, 2018).

Ao longo dos anos, vários autores propuseram outros modelos para explicar o desenvolvimento e a manutenção dos comportamentos excessivos relacionados com o uso da internet, tais como o modelo do anonimato, da conveniência e do escape (ACE) desenvolvido para avaliar a adição cybersexual (Young, Griffin-Shelley, Cooper, O'Mara, \& Buchanan, 2000), o modelo de fases do uso patológico da internet de Grohol (2017) e o modelo compreensivo do desenvolvimento e manutenção da adição à internet de Winkler e Dörsing (como citado por Cash, Rae, Steel, $\&$ Winkler, 2012). Mais recentemente, foi desenvolvido o modelo neurobiológico-centralizado proposto por Brand, Young e Laier (2014) para tentar explicar as perturbações relacionadas com a internet. Mais recentemente, este modelo foi atualizado para melhor definir a ideia de que todos os comportamentos aditivos são desenvolvidos em consequência da interação entre fatores de risco, respostas afetivas e cognitivas a estímulos específicos, bem como funções executivas (controle inibitório a tomada de decisão) (Brand et al., 2019). Contudo, este modelo carece de validação empírica (Pontes, Kuss, \& Griffiths, 2015). Em relação à avaliação da adição à internet, um dos instrumentos psicométricos mais populares é o Internet Addiction Test (IAT) de Young (1998), tendo sido conduzidos estudos de validade factorial, de construto, convergente e discriminante na população portuguesa, por Pontes, Patrão e Griffiths (2014).

Porém, apesar da falta de consenso que existe, esta adição à internet nada tem a ver com o que realmente determina as dependências (tolerância, dependência, síndrome de abstinência etc.) e os critérios de diagnóstico propostos para que a perturbação de adição fosse incluída nos diagnósticos formais do Manual de Diagnóstico e Estatística das Perturbações Mentais (DSM) também não têm sido admitidos, nem pela Associação Americana de Psiquiatria, nem pela Organização Mundial da Saúde apenas foi incluída a perturbação de jogos de internet com sugestão de estudos futuros (American Psychiatric Association, 2013). De acordo com Starcevic (2013), este conceito de adição enfrenta dois tipos de desafios - o primeiro é sobre isso ser uma adição e o segundo diz respeito à internet como uma via à qual um indivíduo está presumivelmente viciado. Ou seja, vários autores (e.g. Griffiths \& Szabo, 2014; Pontes \& Patrão, 2014; Pontes, Szabo, \& Griffiths, 2015) consideram que o termo "adição" seria mais concreto e adequado para se referir à atividade específica entendida como viciante (por exemplo, dependência de jogos de vídeo on-line). Assim sendo, a nomenclatura existente e frequentemente utilizada para definir o fenómeno apresenta problemas conceptuais resultantes das diferentes posições teóricas existentes. Além disso, a evidência empírica sugere que o termo "adição à internet" deveria ser substituído por outro 
termo que reflita vícios em atividades on-line específicas (Pontes, Szabo, \& Griffiths, 2015).

Posto isto, podemos afirmar que a adição à internet parece estar relacionada com usos específicos de algumas funcionalidades da internet e não com seu uso generalizado. Isto é, os utilizadores não são "viciados" na internet, mas sim em uma ou várias atividades on-line específicas (Billieux, 2012). Além da adição à internet, outras adições cibernéticas são frequentes (Billieux, 2012), como a "adição aos vídeojogos on-line" (Billieux et al., 2011), "adição ao jogo patológico on-line" (Griffiths, 2003), "adição ao sexo on-line" (Meerkerk, Van den Eijnden, \& Garretsen, 2006) e "adição às redes sociais" (Wilson, Fornasier, \& White, 2010). Todas estas adições acarretam consequências negativas para a vida do indivíduo e apresentam fatores de risco comuns (Billieux, 2012). Assim sendo, a adição à internet deveria ser conceituada dentro de uma gama mais ampla de "adições cibernéticas" que passa por comportamentos que dependem de atividades on-line específicas e/ou atividades que envolvem comunicação entre indivíduos através de dispositivos tecnológicos (Billieux, 2012).

Outro elemento discutível associado à adição à internet é que, ao contrário das dependências, a internet oferece múltiplos benefícios e, como tal, não deve ser vista como um dispositivo a ser criticado como "viciante": (1) a internet possibilita rapidez na comunicação, possui carácter interativo e é um apoio à aprendizagem (Spizzirri, Wagner, Mosmann, \& Armani, 2012); (2) a internet tornou-se um importante contexto social para os mais velhos, pois influencia seu bem-estar, isto é, ao usarem a internet aumentam os níveis percebidos de apoio social, reduzem a solidão, melhoram a satisfação com a vida e melhoram seu bem-estar psicológico (Heo, Chun, Lee, Lee, \& Kim, 2015); (3) os estudantes universitários, em particular, podem obter diversos benefícios com o uso da internet para propósitos educacionais (Rayan et al., 2017) - acesso a periódicos on-line, aprendizagem de idiomas, pesquisa académica, navegação em bibliotecas virtuais (Al-Gamal, Alzayyat, \& Ahmad, 2016) - e, também para fins relacionais - navegação na rede de lazer, socialização on-line e, até mesmo, estabelecimento de relacionamentos-(Jones, Johnson-Yale, Millermaier, \& Pérez, 2009); (4) a internet pode ser vista como um meio importante para aumentar a satisfação com a vida entre cidadãos e grupos sociais mais frágeis - pessoas de baixos níveis económicos e/ou pessoas que sofram de problemas de saúde que interferem com o normal funcionamento do seu dia-a-dia (Lissitsa \& ChachashviliBolotin, 2016); (5) pode ser usada como um meio para aumentar o apoio social percebido, como por exemplo, através do Facebook, o que por sua vez diminui os níveis de estresse e aumenta o bem-estar psicológico. Ou seja, a internet pode ser vista como um benefício indireto para a saúde (Wiederhold, 2017). No geral, ela é uma ferramenta tecnológica altamente difundida que torna a deteção e o diagnóstico de adição difíceis (Young, 2004).
São também numerosas as críticas que têm sido apontadas a este conceito: (1) falta de especificidade teórica e conceito de dependência - não dá importância ao que as pessoas estão realmente a fazer quando estão on-line (Caplan, 2002); (2) falta de consenso internacional sobre o conceito e o diagnóstico (Griffiths, Kuss, Billieux, \& Pontes, 2016); (3) falta de esclarecimento de questões específicas sobre a adição à internet que incluem três áreas problemáticas estreitamente relacionadas - terminologia, conceções diagnósticas e medidas (Demetrovics, Szeredi, \& Rózsa, 2008; Tokunaga, 2015); (4) grande parte das pesquisas realizadas acerca da adição à internet são estudos exploratórios, que recorrem a amostras auto-selecionadas e não apresentam grupos de controlo (DeAngelis, 2000; Tokunaga, 2015); (5) alguns investigadores consideram que talvez as consequências pessoais, profissionais e sociais atribuídas a este comportamento possam, na verdade, ser apenas sintomas de outras perturbações ou problemas psicológicos primários (Pies, 2009; Shaffer, Hall, \& Vander Bilt, 2000); e (6) as investigações publicadas carecem de aproximações teóricas de referência e não conseguem determinar relações causais entre as consequências descritas e a utilização da internet caindo no erro de "ignorar a causa comum" (Grohol, 2017).

Em conclusão, falar em adição à internet é equivocado e a expressão deve ser abandonada, apesar de se ter tornado um termo amplamente utilizado (Pontes et al., 2016). Acontece que, atualmente, existe uma tendência para "sobrepatologizar" no que respeita aos comportamentos aditivos o que poderá levar a uma avaliação duvidosa da pesquisa acerca da dependência comportamental e a negligenciar os principais processos psicológicos (Billieux, Schimment, Khazaal, Maurage, \& Heeren, 2015). Surge, então, uma necessidade de investigar mais sobre a dependência comportamental e passar de uma abordagem baseada em critérios para uma focada nos processos psicológicos envolvidos-motivacional, afetivo, cognitivo, interpessoal e social (Billieux et al., 2015). Posto isto, o uso da internet deve deixar de ser visto como uma doença - paradigma de patologia - e passar a ser relacionado com hábitos de autorregulação - paradigma cognitivo-comportamental (Pontes et al., 2016).

\section{Uso problemático da internet}

Na sequência do referido anteriormente, é então que surge o conceito de uso problemático da internet (UPI) que, segundo Tokunaga (2015), foi adotado por grande parte dos investigadores que utilizam o modelo cognitivocomportamental desenvolvido por Davis (2001) e o modelo sociocognitivo do uso da internet não regulamentada desenvolvido por LaRose, Lin e Eastin (2003). De acordo com estas perspetivas, o UPI não é visto como uma doença, patologia ou perturbação clínica (Pontes et al., 2016), mas sim como um padrão distinto de cognições e comportamentos que originam resultados negativos para a vida (Assunção \& Matos, 2017). Tokunaga (2015) refere ainda que o UPI se situa na faixa média do continuum da severidade do problema e é 
de natureza benigna, enquanto a adição à internet se situa na extremidade superior do continuum exigindo a experiência de graves consequências negativas para a vida.

O conceito "uso problemático da internet" foi proposto por Beard e Wolf (2001), sendo que investigadores como Caplan e Davis também o adotaram. Este termo surgiu para definir o uso da internet que provoca, na vida das pessoas, dificuldades psicológicas, sociais, académicas e/ou profissionais. Para estes autores, o termo "problemático" é mais adequado, pois apresenta menos discordâncias teóricas do que outras terminologias. Por outras palavras, o UPI é uma síndrome multidimensional constituída por sintomas cognitivos e comportamentais que resultam em problemas sociais, académicos ou profissionais (Caplan, 2002). Shapira et al. (2003), por sua vez, definem o UPI como uma preocupação mal-adaptativa com o uso da internet que causa sofrimento significativo e/ou prejuízo. É ainda relevante acrescentar que alguns investigadores entendem o UPI como uma forma de procura de reafirmação e/ou um comportamento de evitamento que se destina a reduzir emoções negativas (Wan \& Chiou, 2006). Para além de estar destinado a reduzir emoções negativas, pode estar associado a um maior sofrimento subjetivo, comprometimento funcional e perturbações psiquiátricas (Shapira, Goldsmith, Keck, Khosla, \& McElroy, 2000). Em suma, o UPI poderia ser caracterizado pela incapacidade do indivíduo em controlar o uso da internet, que por sua vez, conduz a sentimentos de angústia e ao comprometimento das atividades diárias (Shapira et al., 2000).

De acordo com a literatura, o UPI pode ainda ser definido como específico ou generalizado. De acordo com Davis (2001), o específico se refere ao uso excessivo de funções específicas de conteúdos da internet, tais como jogar ou ver material on-line de cariz sexual, mas estas problemáticas comportamentais podem manifestar-se de forma alternativa se o indivíduo for incapaz de aceder à internet. Por outro lado, Davis (2001) conceptualiza o generalizado como um uso nãoespecífico e multidimensional da internet que resulta em consequências negativas para o indivíduo. Manifestações do UPI generalizado incluem cognições e comportamentos mal-adaptativos relacionados ao uso da internet que não sejam específicos. Ou seja, a internet é, nestes casos, usada como um veículo para múltiplos propósitos.

Como já foi referido anteriormente, Davis (2001) desenvolveu o modelo cognitivo-comportamental do UPI que sugere que os indivíduos que sofrem de problemas psicossociais são mais propensos a desenvolver UPI. Este modelo menciona que esse fenómeno está estreitamente relacionado com cognições problemáticas, sendo também associado a comportamentos que mantêm ou aumentam estas cognições desajustadas, resultando em consequências negativas para o indivíduo (Davis, 2001). O ponto central deste modelo prende-se com as cognições mal-adaptativas, que emergem por haver uma disfunção cognitiva, podendo ser acerca de si próprio e/ou do mundo, sendo, segundo o autor, suficientes para o aparecimento do UPI. As primeiras dizem respeito a uma visão negativa de si próprio, conduzindo a uma procura de respostas positivas pelos outros, de uma forma não temível, através da internet (Davis, 2001). As segundas, relacionam-se pelo facto do indivíduo considerar que a internet é o único local em que é amado e respeitado pelos outros (Davis, 2001).

As respostas positivas que resultam do estar on-line reforçam o comportamento do indivíduo, aumentando, assim, a probabilidade de nova ocorrência. Uma vez que houve reforço, o indivíduo torna-se condicionado a realizar a atividade mais constantemente de maneira a atingir a mesma resposta que no acontecimento inicial (Davis, 2001).

Os sintomas cognitivos e comportamentais do UPI parecem estar especialmente relacionados com a interação social on-line. Além disso, Caplan (2010) reconheceu que um indivíduo que sofra de problemas psicossociais tenderá a desenvolver perceções negativas das suas competências sociais, que o levarão à preferência pelo estabelecimento de interações sociais on-line, ao invés das tradicionais face-a-face. Esta preferência poderá levar o indivíduo a se autorregular de forma deficiente no que toca à utilização que faz da internet (através do aumento da preocupação cognitiva por estar on-line e uso compulsivo dela) e a utilizá-la para regular o humor (o que, por sua vez, aumentará a autorregulação deficiente). Esta autorregulação deficiente manifestar-se-á, emúltima instância, em consequências negativas em diversos níveis da vida do indivíduo (e.g., económico, académico/ laboral, familiar, social etc.). Por outras palavras, Caplan (2010) reconhece constructos cognitivos e comportamentais que se relacionam com as consequências negativas que estão associadas ao uso da internet - preferência pela interação social on-line; regulação de humor; autorregulação deficiente; preocupação cognitiva e comportamento compulsivo.

Posto isto, e numa tentativa de avançar na conceituação do UPI, Caplan (2010) procurou esclarecer as construções cognitivo-comportamentais de Davis (2001), desenvolvendo dois instrumentos psicométricos baseados na teoria de Davis (2001) para avaliar o UPI generalizado - Generalized problematic internet use scale (GPIUS) (Caplan, 2002) e Generalized problematic internet use scale 2 (GPIUS2) (Caplan, 2010; os estudos de validade factorial, de construto, convergente e discriminante na população portuguesa foram realizados por Pontes et al., 2016).

Importa ainda referir que, em muitas investigações, o uso problemático da internet está relacionado com a presença de várias comorbilidades, tais como perturbações do humor, de uso de substâncias, de ansiedade, de controlo dos impulsos e de personalidade. Assim como com a presença de vários fatores de risco, tais como, idade, sexo masculino (Tsai et al., 2009), falta de suporte emocional (Griffiths, 2015), funcionamento familiar deficitário (Wartberg, Kriston, Kammerl, Petersen, \& Thomasius, 2015), défice de habilidades sociais (Caplan, 2005), isolamento social (Tokunaga, 2015), bem-estar emocional mais pobre (Piguet et al, 2015) e fraco desempenho académico (Boubeta, Ferreiro, Salgado, \& Couto, 2015). Posto isto, não é possível definir ainda, fruto da escassez de estudos longitudinais 
(cf. Tokunaga, 2014), se o uso problemático da internet é a perturbação primária ou se está associado a outras patologias (Carli et al., 2013; Echeburúa, 2000).

\section{Em conclusão: que termo usar?}

O termo "uso problemático da internet" pode ser considerado o mais adequado por duas razões: em primeiro lugar, estamos de acordo com Beard e Wolf (2001) e Caplan (2002) que adotam que a perspetiva da adição ("vício") é inadequada por falta de clareza conceptual e continua a ser discutível se o uso excessivo de internet constitui um vício; em segundo lugar, comparado a "patológico" ou "inadaptado", o termo "problemático" descreve o comportamento de forma mais abrangente, cobrindo toda a gama de comportamentos problemáticos - de comportamentos leves a severamente perturbados (Ang, Chong, Chye, \& Huan, 2012).

Além disso, a perspectiva cognitivocomportamental utilizada na definição do UPI apresenta maior flexibilidade e valor clínico ao contemplar um continuum de severidade em relação ao uso excessivo da internet, permitindo um maior entendimento por parte dos agentes de saúde mental sobre a forma e intensidade como o UPI pode afetar os diversos domínios da vida dos utilizadores problemáticos.
Embora este estudo de revisão tenha concluído que o termo "uso problemático da internet" seja o mais adequado para descrever e caracterizar o fenómeno em análise, tal conclusão pode ser interpretada como potencialmente limitada por não resultar de uma análise proveniente de dados empíricos, mas sim de um processo dedutivo em função da literatura analisada. Deste modo, sugere-se que estudos futuros investiguem com sistematicidade a adequação desta conclusão a nível empírico. Por exemplo, uma vez que o UPI encontra-se associado a atividades on-line específicas, tendo portanto, um foco (Griffiths \& Szabo, 2014; Pontes, Szabo, \& Griffiths, 2015), torna-se pertinente explorar de que modo o uso de aplicações e funcionalidades específicas contribuem para o uso excessivo e potencialmente problemático da internet. Neste sentido, estudos com design experimental poderão ser úteis na exploração e aprofundamento sobre a natureza dos comportamentos aditivos on-line. De igual modo, futuros estudos que utilizem dados comportamentais (behavioral data) poderão ser benéficos no cumprimento deste objetivo e na clarificação sobre a distinção entre o uso normal, uso excessivo e uso problemático da internet, uma vez que a literatura existente não fornece uma resposta cabal a esta questão.

\title{
Internet addiction or problematic internet use? Which term should be used?
}

\begin{abstract}
Internet use has increased exponentially worldwide. Although the use itself is not negative, since it integrates several benefits, some individuals seem to show problems related to its excessive, uncontrolled, and dysfunctional use. Therefore, the interest of researchers in exploring this use, when it is excessive and unhealthy, has been growing, especially in the last two decades. However, being a subject/construct treated by different authors with different theoretical frameworks, several terms are used in the literature to describe this phenomenon. Regarding this, this article proposes to present a literature review of two of the most used and shared concepts in the scientific literature, that is, internet addiction and problematic internet use.
\end{abstract}

Keywords: internet addiction, problematic internet use, conceptualization.

\section{Dépendance à Internet ou utilisation problématique d'Internet? Quel terme utiliser?}

Résumé : L'utilisation d'Internet a augmenté de manière exponentielle dans le monde. Bien que l'utilisation d'Internet ne soit pas négative en soi, étant donné qu'elle intègre plusieurs avantages, certaines personnes semblent présenter des problèmes liés à son utilisation excessive, incontrôlée et dysfonctionnelle. En conséquence, l'intérêt des chercheurs pour exploiter cet usage, qu'il soit excessif ou malsain, a augmenté, particulièrement au cours des deux dernières décennies. Cependant, étant un thème/construit traité par différents auteurs avec différents cadres théoriques, plusieurs termes sont utilisés dans la littérature pour décrire ce phénomène. En ce sens, nous proposons de présenter dans cet ouvrage une analyse de deux des concepts les plus utilisés et les plus partagés dans la littérature scientifique, à savoir l'addition à internet et son utilisation problématique.

Mots-clés : ajout à l'Internet, utilisation d'Internet problématique, conceptualisation.

\section{Adición a Internet o uso problemático de Internet: ¿Qué término usar?}

Resumen: El uso de Internet ha aumentado exponencialmente a nivel mundial. Aunque esto no es por sí solo negativo ya que integra diversos beneficios, algunos individuos parecen presentar problemas relacionados con su uso excesivo, descontrolado 
y disfuncional. En consecuencia, en las últimas dos décadas ha aumentado cada vez más el interés de los investigadores en estudiar este uso, cuando es excesivo o poco saludable. Sin embargo, y siendo un tema/constructo tratado por diferentes autores con cuadros teóricos también diferentes, son varios los términos usados en la literatura para describir este fenómeno. En este sentido, nos proponemos presentar en este trabajo una revisión de dos de los conceptos más usados y más divididos en la literatura científica, es decir, adición a Internet y uso problemático de Internet.

Palabras clave: adición a Internet, uso problemático de Internet, conceptualización.

\section{Referências}

Al-Gamal, E., Alzayyat, A., \& Ahmad, M. M. (2016). Prevalence of internet addiction and its association with psychological distress and coping strategies among university students in Jordan. Perspectives in Psychiatric Care, 52(1), 49-61. doi: 10.1111/ppc.12102

American Psychiatric Association. (2013). Diagnostic and statistical manual of mental disorders (5a. ed.). Arlington: American Psychiatric Association.

Ang, R. P., Chong, W. H., Chye, S., \& Huan, V. S. (2012). Loneliness and generalized problematic internet use: parents' perceived knowledge of adolescents' online activities as a moderator. Computers in Human Behavior, 28(4), 1342-1347. doi:10.1016/j.chb.2012.02.019

Assunção, R. S., \& Matos, P. M. (2017). The generalized problematic internet use scale 2: validation and test of the model to Facebook use. Journal of Adolescence, 54, 51-59. doi: 10.1016/j.adolescence.2016.11.007

Beard, K. W., \& Wolf, E. M. (2001). Modification in the proposed diagnostic criteria for internet addiction. CyberPsychology \& Behavior, 4(3), 377-383. doi:10.1089/109493101300210286

Billieux, J. (2012). Problematic use of the mobile phone: a literature review and a pathways model. Current Psychiatry Reviews, 8(4), 299-307. doi: 10.2174/157340012803520522

Billieux, J., Chanal, J., Khazaal, Y., Rochat, L., Gay, P., Zullino, D., \& Van der Linden, M. (2011). Psychological predictors of problematic involvement in massively multiplayer online role-playing games: illustration in a sample of male cybercafé players. Psychopathology, 44(3), 165-171.

Billieux, J., Schimmenti, A., Khazaal, Y., Maurage, P., \& Heeren, A. (2015). Are we overpathologizing everyday life? A tenable blueprint for behavioral addiction research. Journal of Behavioral Addictions, 4(3), 119123. doi: 10.1556/2006.4.2015.009

Brand, M., Wegmann, E., Stark, R., Müller, A., Wölfling, K., Robbins, T. W., \& Potenza, M. N. (2019). The Interaction of Person-Affect-Cognition-Execution (I-PACE) model for addictive behaviors: update, generalization to addictive behaviors beyond internetuse disorders, and specification of the process character of addictive behaviors. Neuroscience and Biobehavioral Reviews, 104, 1-10. doi: 10.1016/j. neubiorev.2019.06.032
Brand, M., Young, K. S., \& Laier, C. (2014). Prefrontal control and internet addiction: a theoretical model and review of neuropsychological and neuroimaging findings. Frontiers in Human Neuroscience, 8, 375. doi: 10.3389/fnhum.2014.00375

Boubeta, A. R., Ferreiro, S. G., Salgado, P. G., \& Couto, C. B. (2015). Variables asociadas al uso problemático de internet entre adolescentes. Health and Addictions, 15(1), 25-38.

Cabral, F., Pereira, M., \& Teixeira, C. M. (2018). Internet, physical activity, depression, anxiety and stress. PsychTech \& Health Journal, 2(1), 15-27. doi: 10.26580/ PTHJ.art10-2018

Caplan, S. E. (2002). Problematic internet use and psychosocial well-being: development of a theorybased cognitive-behavioral measurement instrument. Computers in Human Behavior, 18(5), 553-575. doi:10.1016/S0747-5632(02)00004-3

Caplan, S. E. (2005). A social skill account of problematic internet use. Journal of Communication, 55(4), 721-736. doi: $10.1111 / \mathrm{j} .1460-2466.2005 . t b 03019 . x$

Caplan, S. E. (2010). Theory and measurement of generalized problematic internet use: a two-step approach. Computers in Human Behavior, 26(5), 1089-1097. doi:10.1016/j. chb.2010.03.012

Carbonell, X., Fúster, H., Chamarro, A., \& Oberst, U. (2012). Adicción a internet y móvil: una revision de estudios empíricos españoles. Papeles del Psicólogo, 33(2), 82-89.

Carli, V., Durkee, T., Wasserman, D., Hadlaczky, G., Despalins, R., Kramarz, E., et al. (2013). The association between pathological internet use and comorbid psychopathology: a systematic review. Psychopathology, 46(1), 1-13. doi: 10.1159/000337971

Cash, H., Rae, C. D., Steel, A. H., \& Winkler, A. (2012). Internet addiction: a brief summary of research and practice. Current Psychiatry Reviews, 8(4), 292-298. doi: $10.2174 / 157340012803520513$

Chen, K., Tarn, J. M., \& Han, B. T. (2004). Internet dependency: its impact on online behavioral patterns in e-commerce. Human Systems Management, 23(1), 49-58.

Davis, R. A. (2001). A cognitive-behavioral model of pathological INTERNET use. Computers in Human Behavior, 17(2), 187-195. doi: 10.1016/S07475632(00)00041-8 
DeAngelis, T. (2000). Is internet addiction real? Monitor on Psychology, 31(4), 1-5.

Demetrovics, Z., Szeredi, B., \& Rózsa, S. (2008). The threefactor model of internet addiction: the development of the problematic internet use questionnaire. Behavior Research Methods, 40(2), 563-574. doi: 10.3758/ BRM.40.2.563

Echebúria. E. (2000). Medicíon clínica en transtornos fóbicos y de ansiedade. In A. Bulbena Vilarrasa, G. Berrios, \& P. Fernández de Larrinoa Palacios (Orgs.), Medición Clínica en Psiquiatria y Psicologia (pp. 369375). Barcelona: Masson.

Echeburúa, E., \& Corral, P. (2010). Adicción a las nuevas tecnologías y a las redes sociales en jóvenes: un nuevo reto. Adicciones, 22(2), 91-95. doi: 10.20882/ adicciones. 196

Elhai, J. D., Dvorak, R. D., Levine, J. C., \& Hall, B. J. (2017). Problematic smartphone use: a conceptual overview and systematic review of relations with anxiety and depression psychopathology. Journal of Affective Disorders, 207, 251-259. doi: 10.1016/j.jad.2016.08.030

Garcia Del Castillo, J. A. (2013). Adicciones tecnológicas: el auge de las redes sociales. Salud y Drogas, 13(1), 5-13.

Griffiths, M. D. (1995). Technological addictions. Clinical Psychology Forum, 76, 14-19.

Griffiths, M. (2003). Internet gambling: issues, concerns, and recommendations. CyberPsychology \& Behavior, 6(6), 557-568. doi: 10.1089/109493103322725333

Griffiths, M. D. (2005). A “components” model of addiction within abiopsychosocial framework. Journal of Substance Use, 10(4), 191-197. doi: 10.1080/14659890500114359

Griffiths, M. D. (2015). Classification and treatment of behavioural addictions. Nursing in Practice, 82, 44-46.

Griffiths, M. D., Kuss, D. J., Billieux, J., \& Pontes, H. M. (2016). The evolution of internet addiction: a global perspective. Addictive Behaviors, 53, 193-195. doi: 10.1016/j.addbeh.2015.11.001

Griffiths, M. D., \& Szabo, A. (2014). Is excessive online usage a function of medium or activity? An empirical pilot study. Journal of Behavioral Addictions, 3(1), 7477. doi: 10.1556/JBA.2.2013.016

Grohol, J. M. (2017). Internet addiction guide. Retrieved from https://psychcentral.com/netaddiction/

Heo, J., Chun, S., Lee, S., Lee, K. H., \& Kim, J. (2015). Internet use and well-being in older adults. Cyberpsychology, Behavior, and Social Networking, 18(5), 268-272. doi: 10.1089/cyber.2014.0549

Instituto Nacional de Estatística. (2014). Mais de metade dos utilizadores da internet fazem-no em mobilidade. Retrieved from http://bit.ly/2YTeB7W

Internet World Stats (2017). 2017 World stats. Retrieved from http://www.internetworldstats.com/

Jones, S., Johnson-Yale, C., Millermaier, S., \& Pérez, F. S. (2009). US college students' internet use: race, gender and digital divides. Journal of Computer-Mediated Communication, 14(2), 244-264. doi: 10.1111/j.10836101.2009.01439.x
King, D., Delfabbro, P., \& Griffiths, M. (2012). Clinical interventions for technology-based problems: excessive internet and video game use. Journal of Cognitive Psychotherapy, 26(1), 43-56. doi: 10.1891/08898391.26.1.43

Kuss, D., \& Griffiths, M. (2017). Social networking sites and addiction: ten lessons learned. International Journal of Environmental Research and Public Health, 14(3), E311. doi: 10.3390/ijerph14030311

LaRose, R., Lin, C. A., \& Eastin, M. S. (2003). Unregulated internet usage: addiction, habit, or deficient selfregulation? Media Psychology, 5(3), 225-253. doi: 10.1207/S1532785XMEP0503_01

Lissitsa, S., \& Chachashvili-Bolotin, S. (2016). Life satisfaction in the internet age: changes in the past decade. Computers in Human Behavior, 54, 197-206. doi: 10.1016/j.chb.2015.08.001

Marktest (2016). 5,6 milhões de utilizadores de internet em Portugal. Retrieved from http://www.marktest.com/wap/ a/n/id 209b.aspx

Meerkerk, G. J., Van den Eijnden, R. J. V. D., \& Garretsen, H. F. (2006). Predicting compulsive internet use: it's all about sex! CyberPsychology \& Behavior, 9(1), 95-103. doi: $10.1089 / \mathrm{cpb} .2006 .9 .95$

Mendes, I., \& Silva, I. (2017). Uso problemático da internet em adultos: que relação com sintomas clínicos? Revista de Estudios e Investigación en Psicología y Educación, Extr., (13), 129-133. doi: 10.17979/reipe.2017.0.13.2565

Patrão, I., Reis, J., Madeira, L., Paulino, M. C. S., Barandas, R., Sampaio, D., et al. (2017). Avaliação e intervenção terapêutica na utilização problemática da internet (UPI) em jovens: revisão da literatura. Psicologia da Criança e do Adolescente, 7(1-2), 221-243.

Pies, R. (2009). Should DSM-V designate "internet addiction" a mental disorder? Psychiatry (Edgmont), 6(2), 31-37.

Piguet, C., Berchtold, A., Akre, C., \& Suris, J. C. (2015). What keeps female problematic internet users busy online? European Journal of Pediatrics, 174(8), 10531059. doi: 10.1007/s00431-015-2503-y

Pontes, H., Caplan, S. E., \& Griffiths, M. D. (2016). Psychometric validation of the generalized problematic internet use scale 2 in a Portuguese sample. Computers in Human Behavior, 63, 823-833. doi: 10.1016/j. chb.2016.06.015

Pontes, H. M., Kuss, D. J., \& Griffiths, M. D. (2015). Clinical psychology of internet addiction: a review of its conceptualization, prevalence, neuronal processes, and implications for treatment. Neuroscience \& Neuroeconomics, 4, 11-23. doi: 10.2147/NAN.S60982

Pontes, H. M., \& Patrão, I. M. (2014). Estudo exploratório sobre as motivações percebidas no uso excessivo da internet em adolescentes e jovens adultos. Psychology, Community \& Health, 3(2), 90-102. doi: 10.5964/pch. v3i2.93

Pontes, H. M., Patrão, I. M., \& Griffiths, M. D. (2014). Portuguese validation of the internet addiction test: an 
empirical study. Journal of Behavioral Addictions, 3(2), 107-114. doi: 10.1556/JBA.3.2014.2.4

Pontes, H. M., Szabo, A., \& Griffiths, M. D. (2015). The impact of internet-based specific activities on the perceptions of internet addiction, quality of life, and excessive usage: a cross-sectional study. Addictive Behaviors Reports, 1, 19-25. doi: 10.1016/j.abrep.2015.03.002

Kuss, D. J., \& Pontes, H. M. (2019). Internet addiction (Vol. 41). Boston, MA: Hogrefe.

Potenza, M. N. (2006). Should addictive disorders include non-substance-related conditions? Addiction, 101(Suppl. 1), 142-151.

Rayan, A., Dadoul, A. M., Jabareen, H., Sulieman, Z., Alzayyat, A., \& Baker, O. (2017). Internet use among university students in south West Bank: prevalence, advantages and disadvantages, and association with psychological health. International Journal of Mental Health and Addiction, 15(1), 118-129. doi: 10.1007/ s11469-016-9658-2

Shaffer, H. J., Hall, M. N., \& Vander Bilt, J. (2000). "Computer addiction": a critical consideration. American Journal of Orthopsychiatry, 70(2), 162-168. doi: 10.1037/h0087741

Shapira, N. A., Lessig, M. C., Goldsmith, T. D., Szabo, S. T., Lazoritz, M., Gold, M. S., \& Stein, D. J. (2003). Problematic internet use: proposed classification and diagnostic criteria. Depression and Anxiety, 17(4), 207216. doi: 10.1002/da. 10094

Shapira, N. A., Goldsmith, T. D., Keck, P. E., Khosla, U. M., \& McElroy, S. L. (2000). Psychiatric features of individuals with problematic internet use. Journal of Affective Disorders, 57(1), 267-272. doi: 10.1016/ S0165-0327(99)00107-X

Sharifat, H., Rashid, A. A., \& Suppiah, S. (2018). Systematic review of the utility of functional MRI to investigate internet addiction disorder: recent updates on resting state and task-based fMRI. Malaysian Journal of Medicine and Health Sciences, 14(1), 21-33.

Spizzirri, R. C. P., Wagner, A., Mosmann, C. P., \& Armani, A. B. (2012). Adolescência conectada: mapeando o uso da internet em jovens internautas. Psicologia Argumento, 30(69), 327-335.

Starcevic, V. (2013). Is internet addiction a useful concept? Australian and New Zealand Journal of Psychiatry, 47(1), 16-19. doi: 10.1177/0004867412461693

Starcevic, V., \& Aboujaoude, E. (2017). Internet addiction: reappraisal of an increasingly inadequate concept. CNS Spectrums, 22(1), 7-13. doi: 10.1017/ S1092852915000863

Tsai, H., Cheng, S., Yeh, T., Shih, C., Chen, K., Yang, Y., \& Yang, Y. (2009). The risk factors of internet addiction: a survey of university freshmen. Psychiatry Research, 167(3), 294-299. doi: 0.1016/j.psychres.2008.01.015

Tokunaga, R. S. (2014). A unique problem or the manifestation of a preexistent disorder? The mediating role of problematic internet use in the relationships between psychosocial problems and functional impairment. Communication Research, 41(4), 531-560. doi: 10.1177/0093650212450910

Tokunaga, R. S. (2015). Perspectives on internet addiction, problematic internet use, and deficient self-regulation: contributions of communication research. Annals of the International Communication Association, 39(1), 131161. doi: 10.1080/23808985.2015.11679174

Tokunaga, R. S., \& Rains, S. A. (2016). A review and meta-analysis examining conceptual and operational definitions of problematic internet use. Human Communication Research, 42(2), 165-199. doi: 10.1111/ hcre. 12075

Vivas, G. P. M., \& Torres, F. C. (2011). Internet y pánico moral: revisión de la investigación sobre la interaccíon de niños y jóvenes con los nuevos médios. Universitas Psychologica, 10(3), 855-865.

Wan, C., \& Chiou, W. (2006). Psychological motives and online games addiction: a test of flow theory and humanistic needs theory for Taiwanese adolescents. CyberPsychology and Behavior, 9(3), 317-324. doi: 10.1089/cpb.2006.9.317

Wartberg, L., Kriston, L., Kammerl, R., Petersen, K. U., \& Thomasius, R. (2015). Prevalence of pathological internet use in a representative German sample of adolescents: results of a latent profile analysis. Psychopathology, 48(1), 25-30. doi: 10.1159/000365095

Wiederhold, B. K. (2017). Beyond direct benefits: indirect health benefits of social media use. Cyberpsychology, Behavior, and Social Networking, 20(1), 1-2. doi: 10.1089/cyber.2016.29059.bkw

Wiederhold, B. K. (2018). Stop scrolling, start living: the growing reality of internet addiction disorder. Cyberpsychology, Behavior, and Social Networking, 21(5), 279-280. doi: 10.1089/cyber.2018.29111.bkw

Wilson, K., Fornasier, S., \& White, K. M. (2010). Psychological predictors of young adults' use of social networking sites. Cyberpsychology, Behavior, and Social Networking, 13(2), 173-177.

Young, K. S. (1998). Internet addiction: the emergence of a new clinical disorder. Cyberpsychology \& Behavior, 1(3), 237-244. doi: 10.1089/cpb.1998.1.237

Young, K. S. (2004). Internet addiction: a new clinical phenomenon and its consequences. American Behavioral Scientist, 48(4), 402-415. doi: 10.1177/0002764204270278

Young, K. S., Griffin-Shelley, E., Cooper, A., O'mara, J., \& Buchanan, J. (2000). Online infidelity: a new dimension in couple relationships with implications for evaluation and treatment. Sexual Addiction \& Compulsivity: The Journal of Treatment and Prevention, 7(1-2), 59-74. doi: 10.1080/10720160008400207

Recebido: 21/06/2019 Aprovado: 04/08/2019 